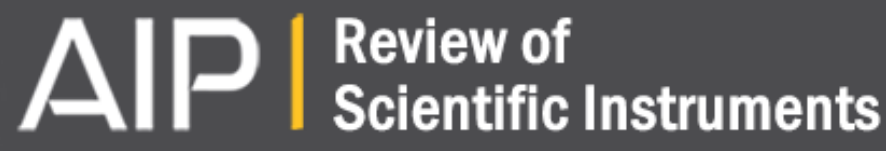

The thirty gigahertz instrument receiver for the Q-U-I Joint Tenerife experiment:

\section{Concept and experimental results}

Enrique Villa, Juan L. Cano, Jaime Cagigas, David Ortiz, Francisco J. Casas, Ana R. Pérez, Beatriz Aja, J.

Vicente Terán, Luisa de la Fuente, Eduardo Artal, Roger Hoyland, and Ángel Mediavilla

Citation: Review of Scientific Instruments 86, 024702 (2015); doi: 10.1063/1.4907015

View online: http://dx.doi.org/10.1063/1.4907015

View Table of Contents: http://scitation.aip.org/content/aip/journal/rsi/86/2?ver=pdfcov

Published by the AIP Publishing

Articles you may be interested in

Polar: Instrument and results

AIP Conf. Proc. 616, 175 (2002); 10.1063/1.1475624

The MAXIMA and MAXIPOL experiments

AIP Conf. Proc. 616, 12 (2002); 10.1063/1.1475596

The low frequency instrument of the Planck mission

AIP Conf. Proc. 609, 144 (2002); 10.1063/1.1471837

The SPOrt experiment

AIP Conf. Proc. 609, 109 (2002); 10.1063/1.1471832

Small scale I, U, Q galaxy noise at $\mathrm{cm}$. waves

AIP Conf. Proc. 609, 51 (2002); 10.1063/1.1471822

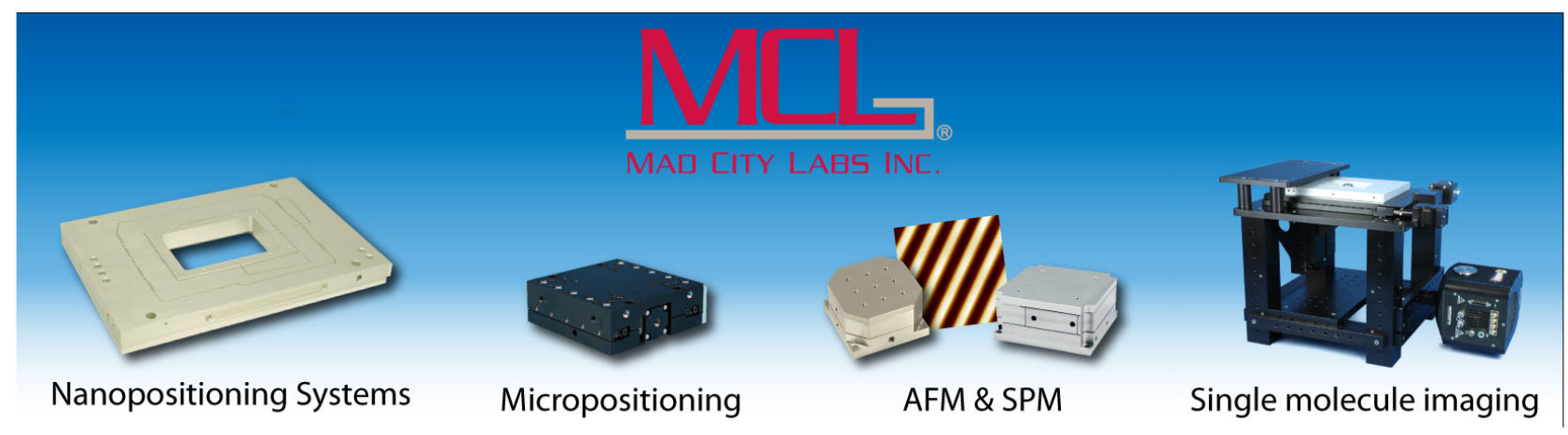




\title{
The thirty gigahertz instrument receiver for the Q-U-I Joint Tenerife experiment: Concept and experimental results
}

\author{
Enrique Villa, ${ }^{1, a)}$ Juan L. Cano, ${ }^{1}$ Jaime Cagigas, ${ }^{1}$ David Ortiz, ${ }^{2}$ Francisco J. Casas, ${ }^{2}$ \\ Ana R. Pérez, ${ }^{1}$ Beatriz Aja, ${ }^{1}$ J. Vicente Terán, ${ }^{1}$ Luisa de la Fuente, ${ }^{1}$ Eduardo Artal, ${ }^{1}$ \\ Roger Hoyland, ${ }^{3}$ and Ángel Mediavilla ${ }^{1}$ \\ ${ }^{1}$ Departamento Ingeniería de Comunicaciones, Universidad de Cantabria, Plaza de la Ciencia s/n, \\ Santander 39005, Spain \\ ${ }^{2}$ Instituto de Física de Cantabria, Avda. Los Castros s/n, Santander 39005, Spain \\ ${ }^{3}$ Instituto de Astrofísica de Canarias, Vía Láctea s/n, La Laguna 38205, Spain
}

(Received 12 December 2014; accepted 19 January 2015; published online 4 February 2015)

\begin{abstract}
This paper presents the analysis, design, and characterization of the thirty gigahertz instrument receiver developed for the Q-U-I Joint Tenerife experiment. The receiver is aimed to obtain polarization data of the cosmic microwave background radiation from the sky, obtaining the $Q, U$, and $I$ Stokes parameters of the incoming signal simultaneously. A comprehensive analysis of the theory behind the proposed receiver is presented for a linearly polarized input signal, and the functionality tests have demonstrated adequate results in terms of Stokes parameters, which validate the concept of the receiver based on electronic phase switching. (O 2015 AIP Publishing LLC. [http://dx.doi.org/10.1063/1.4907015]
\end{abstract}

\section{INTRODUCTION}

The Cosmic Microwave Background (CMB) is the thermal radiation from the Big Bang explosion, which fills the whole Universe. ${ }^{1}$ The existence of the CMB radiation ${ }^{2}$ was postulated by Gamow, Alpher, and Herman in the late 1940s when they were investigating the primordial nucleosynthesis of light elements. However, it was first detected in 1964, when Penzias and Wilson accidentally measured an excess of noiselike signal which could not be removed. ${ }^{3}$ The characterization of the $\mathrm{CMB}$ radiation is, nowadays, one of the most important observational probes in cosmology, as it allows to set constraints on the main cosmological parameters that describe our Universe. Hence, a great effort has been invested over the last decades in developing the necessary technology, in particular radio astronomy receivers, to collect high-sensitivity CMB data. A first space mission dedicated to the analysis of the CMB was the Cosmic Background Explorer (COBE) ${ }^{4}$ in the late 1980s, which measured the CMB anisotropies and whose results were improved by the Wilkinson Microwave Anisotropy Probe (WMAP) ${ }^{4}$ in the early 2000s. More recently, the PLANCK mission ${ }^{5}$ was launched with a wider frequency range, more sensitive receivers, and a higher angular resolution than the previous missions. While the focus of the previous space missions, as well as of many other ground-based or balloon-borne experiments, has been the characterization of the intensity properties of the $\mathrm{CMB}$, nowadays, the interest of the community has shifted to the study of the polarization of the CMB. The reason for this is the search for the primordial $\mathrm{B}$-mode signal that might be encoded in the CMB polarization pattern. This signal may be a proof of inflation, a theoretical framework that explains the evolution of the Universe in

\footnotetext{
a) Author to whom correspondence should be addressed. Electronic mail: villae@unican.es
}

the moment right after the Big Bang. ${ }^{6}$ Among ground-based projects, QUIET $^{7}$ and FARADAY ${ }^{8}$ have developed sensitive radiometers based on the schemes of previous receivers performing high-quality sky maps. A new ground-based polarization experiment, called Q-U-I Joint Tenerife (QUIJOTE) experiment, is under development with the aim of characterizing the polarization of the CMB and other galactic and extragalactic signals at medium and large angular scales in the frequency range from 10 to $47 \mathrm{GHz} .{ }^{9,10}$ The configuration of the receiver enables to obtain $Q, U$, and $I$ Stokes parameters simultaneously, which describe the polarization states of an electromagnetic radiation in terms of the total intensity, the fractional degree of polarization, and the shape parameters of the polarization ellipse. ${ }^{11}$

This paper presents the analysis, design, and characterization of the receiver for the QUIJOTE Thirty-GHz Instrument (TGI), verifying the functionality of the receiver according to the theory. A representative polarimeter is analyzed obtaining the detected voltages in each output which depend on the polarization of the incoming signal. Furthermore, the measurements of the receiver response validate the instrument concept comparing measured values with the theoretical ones. The document is divided into five sections. The first one gives an introduction and, then, the QUIJOTE experiment is described and analyzed in Sec. II. Section III is focused in the subsystems which make up the receiver, giving an overview of their performances. The functionality test is presented and discussed in Sec. IV, and, finally, Sec. V draws general conclusions.

\section{TGI QUIJOTE EXPERIMENT}

The QUIJOTE project is a ground-based experiment installed and being operated at Teide observatory (Canary Islands, Spain) and it is divided into two different stages. In the first one, called Phase I, a multi-frequency instrument (MFI), 
consisting of 4 pixels with 8 channels per horn, characterizes the $\mathrm{CMB}$ covering different bands within the frequency range from 10 to $20 \mathrm{GHz}$. In the Phase II, two instruments are under development for a second telescope, a 30-GHz (TGI-Thirty$\mathrm{GHz}$ Instrument) and a 40-GHz (FGI-Forty-GHz Instrument). The TGI and the FGI instruments are composed of 31 pixels each one working in the $26-36 \mathrm{GHz}$ and the $35-47 \mathrm{GHz}$ frequency band, respectively.

\section{A. Scientific goals}

The main scientific goals of QUIJOTE are to detect the primordial B-mode signal down to $\mathrm{r}=0.05$ and to characterize the polarization of low-frequency Galactic foregrounds, in particular, the synchrotron emission and the anomalous microwave emission. ${ }^{10}$ QUIJOTE data will be a valuable complement of Planck polarization data at higher frequency, which will provide information about the polarization properties of the thermal dust, a foreground that shows up at frequencies above $100 \mathrm{GHz}$.

From previous experiments, ${ }^{12}$ most CMB information has been obtained only from intensity measurements. Therefore, the analysis of its polarization signal provides helpful data in order to completely characterize the CMB radiation.

The standard theory states that the CMB is linearly polarized, so its polarization state can be described using $Q$ and $U$ Stokes parameters, defined by complex spin spherical harmonics. However, scientific polarization maps are usually defined in terms of E- and B-field components, which correspond to a combination of $Q$ and $U$ coefficients. These parameters enable the calculation of the angular power spectra in terms of temperature, E- and B-modes, which define the way in which the CMB anisotropies are originated from scalar or gravitational waves perturbations, respectively.

The QUIJOTE experiment is defined with two polarization surveys. This strategy enables the receiver to scan sky areas and to obtain significant information of inflationary state of the CMB. The first one is a deep survey intended to analyze a sky area of around 3000 square degrees, whereas the second one is a shallow survey scanning around 18000 square degrees. Both surveys will reach low sensitivities in order to obtain sky maps and synchrotron information.

\section{B. TGI receiver block diagram}

The QUIJOTE TGI radiometer is intended to measure the polarization of the CMB measuring three of the Stokes parameters $(Q, U$, and $I)$, simultaneously. They are related to the amplitude of the two orthogonal electrical field components, providing the power of the signal through the $I$ parameter and the linear polarization from the $Q$ and $U$ parameters.

The calculation of the Stokes parameters is achieved from the combination of measurable signals in the receiver. A polarizer, placed in front of an orthomode transducer (OMT), provides left- and right-hand circular polarization output signals, which detected and properly combined enable to obtain the parameters.

The TGI polarimeter block diagram is shown in Fig. 1. Each pixel of the TGI is composed of a cold stage module (20 $\mathrm{K})$ and a room temperature $(298 \mathrm{~K})$ module. The cryogenic part is made up of a feedhorn, a polarizer, an OMT, and two low-noise amplifiers (LNAs). Outside the cryostat, two gain and filtering modules, the phase switches module, and the correlation and detection module operate at room temperature, in which the microwave signal is amplified, filtered, correlated by $180^{\circ}$ microstrip hybrids and, finally, converted into DC voltages using square-law detectors. These signals are collected by a data acquisition system (DAS). The phase switches modules comprise $0^{\circ} / 180^{\circ}$ and $0^{\circ} / 90^{\circ}$ phase switches generating four polarization states and their performance is crucial in order to obtain the Stokes parameters, minimizing the leakage among them and, at the same time, overcoming the $1 / \mathrm{f}$ noise and different systematic errors in the receiver.

\section{TGI receiver analysis}

The sketch in Fig. 1 shows four outputs $\left(V_{d 1}-V_{d 4}\right)$ providing the detected DC voltages, which are combined in the DAS.

Considering a circular coordinate system, the Stokes parameters are defined by

$$
\begin{gathered}
I=\left|E_{l}\right|^{2}+\left|E_{r}\right|^{2}, \\
Q=2 \cdot \operatorname{Re}\left(E_{l}^{*} \cdot E_{r}\right), \\
U=-2 \cdot \operatorname{Im}\left(E_{l}^{*} \cdot E_{r}\right), \\
V=\left|E_{l}\right|^{2}-\left|E_{r}\right|^{2},
\end{gathered}
$$

where $E_{l}$ and $E_{r}$ are the electrical field components in a circular coordinate system. The parameter $V$ is assumed to be $V=0$, since it is the sign of circular polarization and the CMB is considered as linearly polarized, and therefore it is not measured.

A simplified schematic of the TGI receiver, shown in Fig. 2, is assumed in order to analyze the operation of the polarimeter. According to the scheme, the combination of the $90^{\circ}$ - and $180^{\circ}$-phase switches $\left(\Phi_{1}, \Phi_{3}\right.$ and $\Phi_{2}, \Phi_{4}$, respectively) provides four phase states per branch. Hence, sixteen phase states are achieved between both branches of the pixel. Since

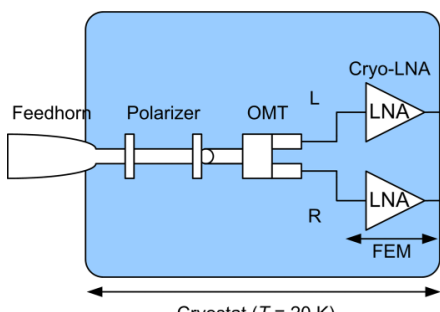

Cryostat $(T=20 \mathrm{~K})$
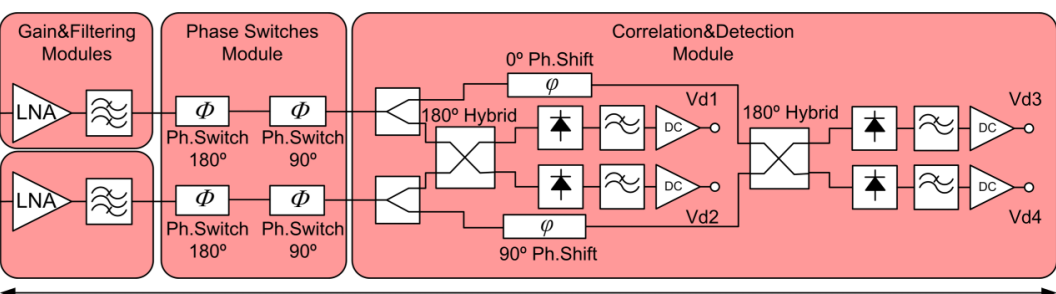

Back-End Module (Room Temperature, $T=298 \mathrm{~K}$ ) 


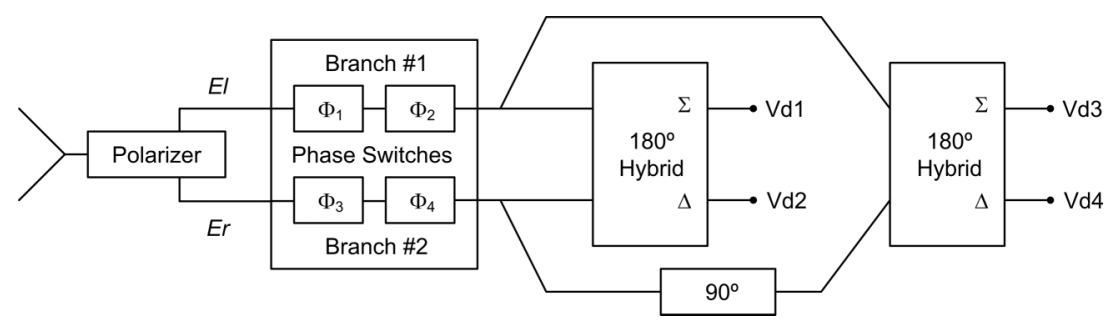

FIG. 2. Simplified sketch of the TGI polarimeter.

different combinations of phase states cause redundant states, the analysis of the phase state of the pixel is easier analyzed in terms of the difference between branches of the pixel $\Phi_{T}$, given by

$$
\Phi_{T}=\Phi_{B 2}-\Phi_{B 1}=\left(\Phi_{3}+\Phi_{4}\right)-\left(\Phi_{1}+\Phi_{2}\right),
$$

where $\Phi_{B 2}$ corresponds to the phase state of the lower branch of the phase switch module and $\Phi_{B 1}$ to the phase state of the upper branch of the phase switch module in Fig. 2.

The input signals to the phase switch module, $E_{l}$ and $E_{r}$, are the outputs of the OMT, which are the components of the incoming electromagnetic radiation to a circular polarization receiver. These signals are defined by

$$
\begin{aligned}
& E_{l} \propto \frac{1}{\sqrt{2}} \cdot\left(E_{X}+j \cdot E_{Y}\right), \\
& E_{r} \propto \frac{1}{\sqrt{2}} \cdot\left(E_{X}-j \cdot E_{Y}\right),
\end{aligned}
$$

where $E_{X}$ and $E_{Y}$ are the orthogonal electrical field components in a Cartesian coordinate system incoming to the feedhorn. Then, considering the phase state $\Phi_{T}=0$ which corresponds to the reference state in each phase switch, the detected output voltages are obtained as

$$
\begin{gathered}
V_{d 1} \propto\left|E_{l}+E_{r}\right|^{2}, \\
V_{d 2} \propto\left|E_{l}-E_{r}\right|^{2}, \\
V_{d 3} \propto\left|E_{l}+j \cdot E_{r}\right|^{2}, \\
V_{d 4} \propto\left|E_{l}-j \cdot E_{r}\right|^{2} .
\end{gathered}
$$

Therefore, performing the linear combination between outputs, the following values are achieved:

$$
\begin{gathered}
V_{d 1}+V_{d 2} \propto\left|E_{l}+E_{r}\right|^{2}+\left|E_{l}-E_{r}\right|^{2}, \\
V_{d 3}+V_{d 4} \propto\left|E_{l}+j \cdot E_{r}\right|^{2}+\left|E_{l}-j \cdot E_{r}\right|^{2}, \\
V_{d 1}-V_{d 2} \propto\left|E_{l}+E_{r}\right|^{2}-\left|E_{l}-E_{r}\right|^{2}=4 \cdot \operatorname{Re}\left(E_{l} \cdot E_{r}^{*}\right), \\
V_{d 3}-V_{d 4} \propto\left|E_{l}+j \cdot E_{r}\right|^{2}-\left|E_{l}-j \cdot E_{r}\right|^{2} \\
=4 \cdot \operatorname{Im}\left(E_{l} \cdot E_{r}^{*}\right) .
\end{gathered}
$$

TABLE I. Stokes parameters from output detected voltages.

\begin{tabular}{lccc}
\hline \hline$\Phi_{\mathrm{T}}$ & $I$ & $Q$ & $U$ \\
\hline $0^{\circ}$ & $V_{d 1}+V_{d 2}=V_{d 3}+V_{d 4}$ & $V_{d 1}-V_{d 2}$ & $V_{d 3}-V_{d 4}$ \\
$90^{\circ}$ & $V_{d 1}+V_{d 2}=V_{d 3}+V_{d 4}$ & $V_{d 3}-V_{d 4}$ & $V_{d 1}-V_{d 2}$ \\
$180^{\circ}$ & $V_{d 1}+V_{d 2}=V_{d 3}+V_{d 4}$ & $V_{d 2}-V_{d 1}$ & $V_{d 4}-V_{d 3}$ \\
$270^{\circ}$ & $V_{d 1}+V_{d 2}=V_{d 3}+V_{d 4}$ & $V_{d 4}-V_{d 3}$ & $V_{d 2}-V_{d 1}$ \\
\hline \hline
\end{tabular}

These values correspond to the Stokes parameters defined in a circular coordinate system, Eq. (1)-(4). In an analogous way, the Stokes parameters are calculated for the other phase states and they are listed in Table I.

An alternative method to measure the Stokes parameters is based on their calculation from the detected voltage values of each independent detector, providing a set of values which corresponds to each detector not from each phase state. The main difference between both methods is in the characterization of the systematic errors, during the calibration process, since they come from different sources.

As mentioned before, the CMB is expected to be weakly linearly polarized. Therefore, the previous analysis is focused on a linear polarization as input signal to the polarimeter. The translation from linear polarization signal to circular polarized wave is performed with the square quad-ridge waveguide polarizer ${ }^{13}$ combined with the OMT, ${ }^{13}$ which splits left-hand and right-hand circular components. The reference plane of the polarizer is rotated $45^{\circ}$ regarding the reference system of the input signal and the OMT in order to accomplish the behavior of a septum polarizer. ${ }^{14}$ The combination of the polarizer plus OMT enables to overcome the septum polarizer bandwidth limitation with a simple arrangement.

Considering the input signal with and amplitude level $A$, the detected outputs for the phase state $\Phi_{T}=0$ are given by

$$
\begin{gathered}
V_{d 1}=K \cdot A^{2}, \\
V_{d 2}=0, \\
V_{d 3}=\frac{K}{2} \cdot A^{2}, \\
V_{d 4}=\frac{K}{2} \cdot A^{2},
\end{gathered}
$$

where $K$ is a constant related to the amplification in the receiver chain.

By changing the phase state of the phase switches module, the values for the different polarization states are listed in Table II.

TABLE II. Detected voltages for an $\mathrm{x}$-axis polarized signal.

\begin{tabular}{lcccc}
\hline \hline$\Phi_{T}$ & $V_{d 1}$ & $V_{d 2}$ & $V_{d 3}$ & $V_{d 4}$ \\
\hline $0^{\circ}$ & $K \cdot A^{2}$ & 0 & $(K / 2) \cdot A^{2}$ & $(K / 2) \cdot A^{2}$ \\
$90^{\circ}$ & $(K / 2) \cdot A^{2}$ & $(K / 2) \cdot A^{2}$ & $K \cdot A^{2}$ & 0 \\
$180^{\circ}$ & 0 & $K \cdot A^{2}$ & $(K / 2) \cdot A^{2}$ & $(K / 2) \cdot A^{2}$ \\
$270^{\circ}$ & $(K / 2) \cdot A^{2}$ & $(K / 2) \cdot A^{2}$ & 0 & $K \cdot A^{2}$ \\
\hline
\end{tabular}


TABLE III. Detected voltages for a y-axis polarized signal.

\begin{tabular}{lcccc}
\hline \hline$\Phi_{T}$ & $V_{d 1}$ & $V_{d 2}$ & $V_{d 3}$ & $V_{d 4}$ \\
\hline $0^{\circ}$ & 0 & $K \cdot A^{2}$ & $(K / 2) \cdot A^{2}$ & $(K / 2) \cdot A^{2}$ \\
$90^{\circ}$ & $(K / 2) \cdot A^{2}$ & $(K / 2) \cdot A^{2}$ & 0 & $K \cdot A^{2}$ \\
$180^{\circ}$ & $K \cdot A^{2}$ & 0 & $(K / 2) \cdot A^{2}$ & $(K / 2) \cdot A^{2}$ \\
$270^{\circ}$ & $(K / 2) \cdot A^{2}$ & $(K / 2) \cdot A^{2}$ & $K \cdot A^{2}$ & 0 \\
\hline \hline
\end{tabular}

In the particular case of a y-axis linear polarization input signal, the analysis of the receiver response is analogous, and the four detected voltages of the polarimeter are listed in Table III.

\section{QUIJOTE POLARIMETER SUBSYSTEMS}

The subsystems of the TGI receiver are individually assembled and characterized in order to validate their individual performances, which were formerly described in Refs. 13 and 15. A summary of the performances of the feedhorn, the polarizer, the OMT, the gain and filtering module, and the detection and correlation module in the $26-36 \mathrm{GHz}$ frequency band is listed in Table IV.
The phase switches module has been, recently, finished with the functionality covered providing the sixteen phase states by combining its two branches. The module is designed in a WR-28 waveguide chassis and includes in each branch a $180^{\circ}$ (Ref. 16) and a $90^{\circ}$ (Ref. 17) phase switches. The module is provided with transistor-transistor logic (TTL) drivers DR65-0109 from MACOM Technology Solutions adding the full-switching capability to the module.

A detailed view of the assembly inside the chassis of the $90^{\circ}$ - and $180^{\circ}$-phase switches is shown in Fig. 3, and different views of the phase switches module are shown in Fig. 4. The measurement results of the module are depicted in Fig. 5 in the frequency range from 24 to $38 \mathrm{GHz}$. The phase states in each branch related to the reference state are shown in Fig. 5(a), while the insertion loss of each phase state is presented in Fig. 5(b).

From the measurements shown in Fig. 5, the mean values in the frequency band of the receiver (from 26 to $36 \mathrm{GHz}$ ) of each phase state are obtained and they are listed in Table V.

\section{QUIJOTE POLARIMETER TEST}

The functionality test carried out to a representative pixel of the QUIJOTE TGI consists of providing a broadband

TABLE IV. Summary of the individual performances of the subsystems (average values in the 26-36 GHz band).

\begin{tabular}{|c|c|c|}
\hline Subsystem & Photograph & Performance \\
\hline Feedhorn antenna & & $\begin{array}{c}\left|\mathrm{S}_{11}\right|<-22 \mathrm{~dB} \\
\text { Directivity }>20 \mathrm{~dB} \\
\text { Cross-polarization }<-40 \mathrm{~dB}\end{array}$ \\
\hline Polarizer & & $\begin{array}{c}\left|\mathrm{S}_{11}\right|_{\mathrm{TE} 11 \mathrm{~s}}<-25 \mathrm{~dB} \\
\left|\mathrm{~S}_{11}\right|_{\mathrm{TE} 11 \mathrm{c}}<-25 \mathrm{~dB} \\
\left|\mathrm{~S}_{21}\right|>-0.2 \mathrm{~dB} \\
\Delta \Phi=90^{\circ} \pm 1^{\circ}\end{array}$ \\
\hline OMT & & $\begin{array}{c}\left|\mathrm{S}_{11}\right|_{\mathrm{TE} 10}<-25 \mathrm{~dB} \\
\left|\mathrm{~S}_{11}\right| \mathrm{TE} 11<-25 \mathrm{~dB} \\
\left|\mathrm{~S}_{21}\right|>-0.15 \mathrm{~dB} \\
\text { Isolation } \\
\Delta \Phi_{\text {Output1-Output2 }} \sim 50 \mathrm{~dB} \\
\text { Output }_{\text {Outp }}=1^{\circ} \pm 0.5^{\circ}\end{array}$ \\
\hline $\begin{array}{l}\text { Gain and filtering } \\
\text { modules }\end{array}$ & & $\begin{array}{c}\left|\mathrm{S}_{11}\right|<-15 \mathrm{~dB} \\
\left|\mathrm{~S}_{22}\right|<-13 \mathrm{~dB} \\
\left|\mathrm{~S}_{21}\right| \sim 33 \mathrm{~dB}\end{array}$ \\
\hline $\begin{array}{l}\text { Correlation and } \\
\text { detection modules }\end{array}$ & & $\begin{array}{c}\left|S_{11}\right|=\left|S_{22}\right|<-10 \mathrm{~dB} \\
\text { Isolation }>10 \mathrm{~dB}\end{array}$ \\
\hline
\end{tabular}




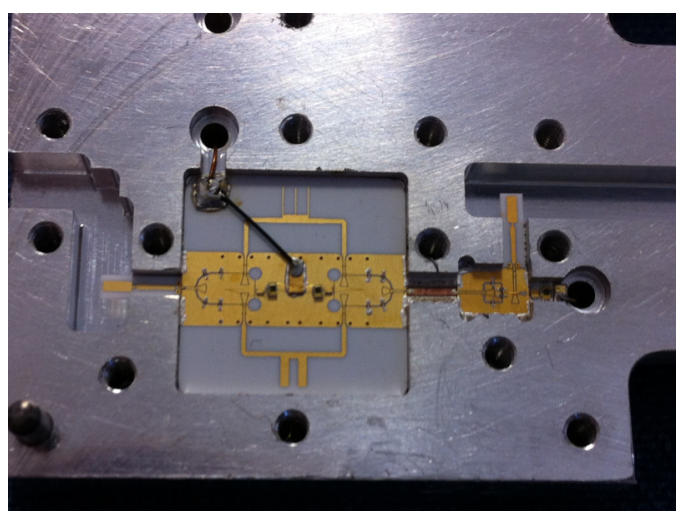

FIG. 3. Detailed view of the cascaded $90^{\circ}$ - and $180^{\circ}$-phase switches.

linearly polarized signal at the receiver input and recording the detected voltages at the Back-End Module (BEM) outputs, expecting Stokes parameters according to the input signal polarization. The receiver chain is fully representative of the pixels that will be installed in the TGI at Teide observatory except the Front-End Module (FEM) cryogenic LNAs which are not included due to the excess of signal power at room temperature. Besides, the functionality of the receiver is not committed by the absence of the cryogenic LNAs since the optomechanics (feedhorn, polarizer, and OMT), phase switches, and detection and correlation modules assure the proper functionality of the receiver. As main consideration,

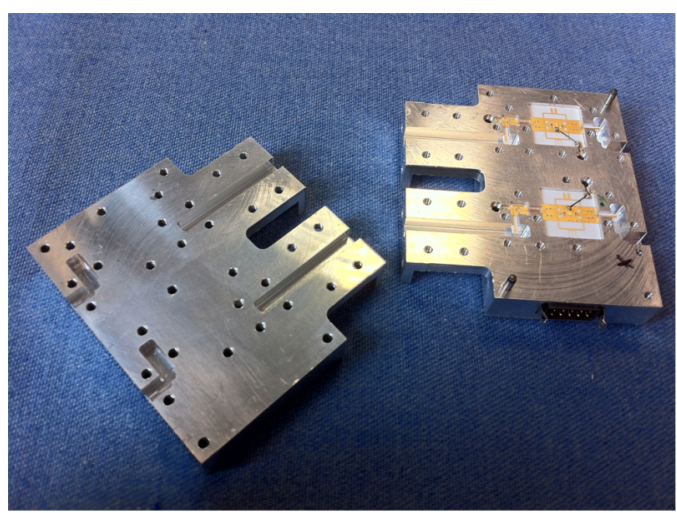

(a)

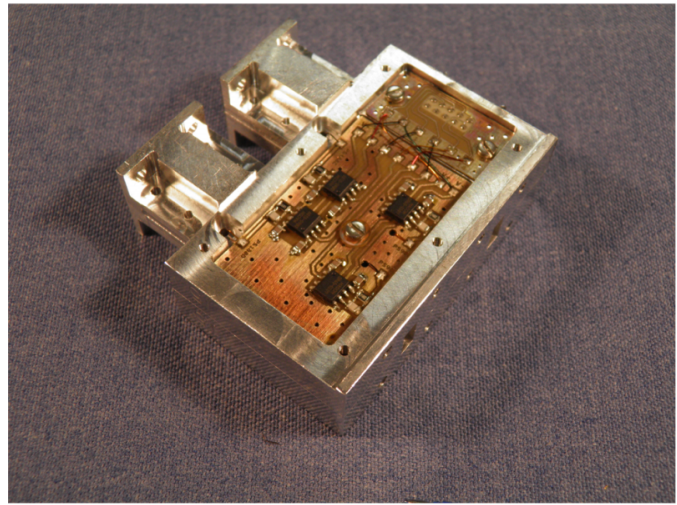

(b)

FIG. 4. Phase switches module. (a) Inside view of the chassis with $180^{\circ}$ and $90^{\circ}$ phase switch circuits. (b) Back view of the chassis with the TTL signals control board.

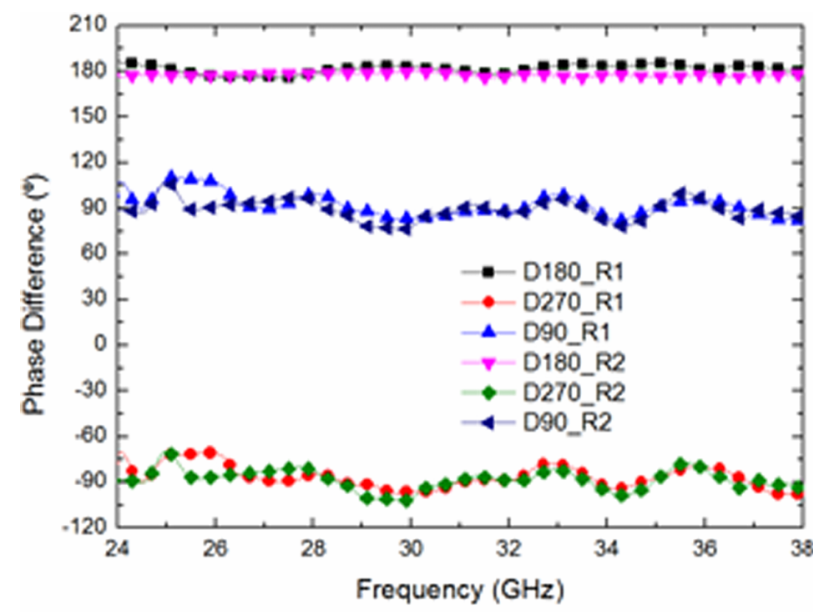

(a)

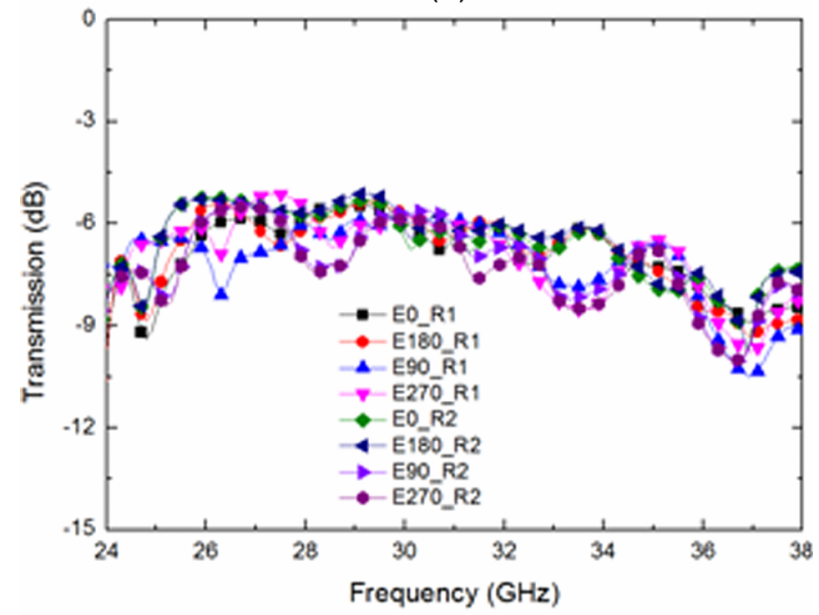

(b)

FIG. 5. Phase switches module results. (a) Phase difference between states for each branch. (b) Transmission coefficient for state and branch. R1 and R2 mean Branch \#1 and Branch \#2, respectively.

the absence of the cryogenic LNAs only implies an analysis of the power budget of the receiver.

The cryogenic LNAs are composed of two cascaded monolithic microwave integrated circuit low-noise amplifiers ${ }^{18}$ with a $5-\mathrm{dB}$ microstrip attenuator allocated between them. A view of the assembly of one module is shown in Fig. 6. The measurement of the cryogenic LNA at $13 \mathrm{~K}$ provides an average noise temperature of $23.1 \mathrm{~K}$ with an average insertion gain of $40.9 \mathrm{~dB}$ for a total power consumption of $7.8 \mathrm{~mW}$ in the 26-36 GHz band. The use of the LNAs at room temperature would provide an excess of power level in the receiver chain which makes the detector operate in its non-linear region. The

TABLE V. Average values of the phase difference in each phase state of the module in the frequency band from 26 to $36 \mathrm{GHz}$.

\begin{tabular}{lc}
\hline \hline State & Phase difference (deg) \\
\hline D180_R1 & 181.06 \\
D270_R1 & -87.73 \\
D90_R1 & 90.74 \\
D180_R2 & 177.73 \\
D270_R2 & -88.88 \\
D90_R2 & 88.71 \\
\hline
\end{tabular}




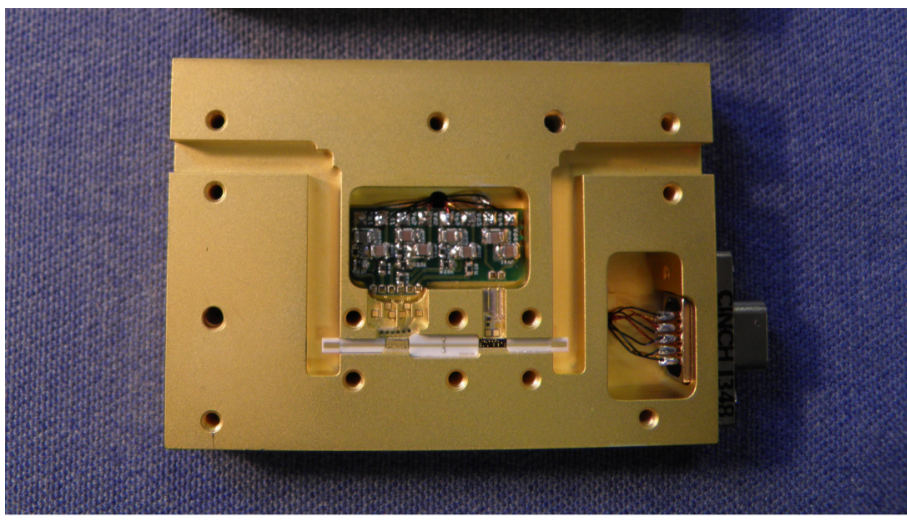

(a)

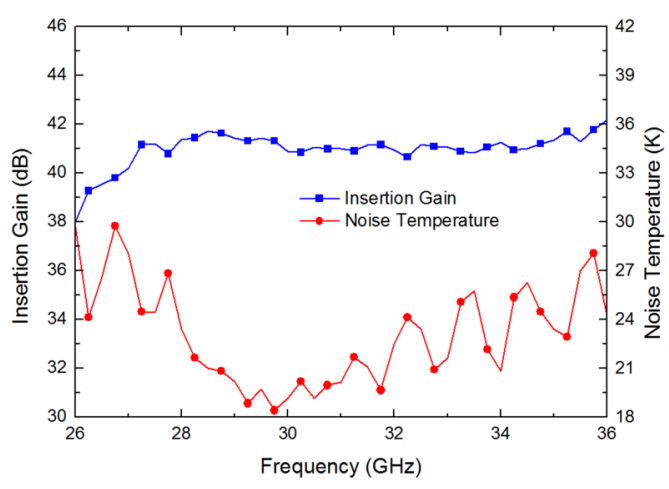

(b)

FIG. 6. Cryogenic low-noise amplifier for the TGI receiver. (a) Assembly. (b) Measurement results.

cryogenic test including the optomechanic parts and the LNAs together with the room temperature subsystems, and using a cold load at the input, will be performed in a dedicated cryostat in the final assembly at the observatory.

Considering the noise results of the representative cryogenic LNA, an equivalent system noise temperature of about $38 \mathrm{~K}$ is expected, since the effect of the optomechanics, spillover effect, and the sky temperature over the bandwidth are directly added to the receiver noise of about $25 \mathrm{~K}$ (addition of the LNA contribution and the rest of the chain).

The test is carried out exciting the receiver with a broadband $\mathrm{x}$-axis linearly polarized signal. A sketch of the functionality test is shown in Fig. 7. Unlike the final application, in the functionality test, it is required a relatively high power polarized signal, much greater than the un-polarized receiver contributions, since the use of a large integration time for data post-processing is beyond the scope of this test. The linear operation of the receiver is assured up to an incoming power level of about $-25 \mathrm{dBm}$ in the microwave detector input, which is composed of a HSCH-9161 Schottky diode and a low-pass resistor-capacitor filter to extract the video signal and shows a narrow window in terms of the power of the incoming signal before working out of its linear region.

The broadband linearly polarized signal is accomplished using a noise source model 346CK01 from Agilent Technologies with an excess noise ratio of $13 \mathrm{~dB}$, which provides an equivalent noise temperature around $6000 \mathrm{~K}$, which is further amplified $(33 \mathrm{~dB})$ and transmitted by a conical horn $(21 \mathrm{dBi}$ of gain). The rectangular waveguide input of the conical horn provides the required linear polarization. The distance between transmitting antenna and the receiver feedhorn is adjusted to avoid compression in the detector devices. The detectors have a sensitivity around $1200 \mathrm{mV} / \mathrm{mW}$, and the gain of the video amplifiers is adjusted to provide output voltages lower than $10 \mathrm{~V}$, according with the DAS and resolution requirements for a 24-bits system PXI-4495 from National Instruments in the telescope.

The two signals split up in the OMT outputs are correlated in the last module of the chain, so the electrical paths of each branch must be identical. Since the individual subsystem in a branch of the receiver could show slight differences in their phase response related to the one assembled in the other branch of the receiver, both signals are affected for a phase imbalance. Therefore, one of the branches of the receiver is provided with an adjusting phase element, which enables the minimization of the phase difference between branches. Thus, the extra electrical length added by the adjusting phase element is compensated by using different lengths in the connection cables from the OMT outputs to the amplifying modules inputs. Hence, the lengths of the flexible coaxial cables are $1 \mathrm{in}$. different.

The detected values are measured covering all the phase switches states, so four TTL signals are implemented to bias

TABLE VI. Actuation TTL control signals for the phase switches.

\begin{tabular}{lcc}
\hline \hline Branch & Phase switch actuation & Frequency $(\mathrm{Hz})$ \\
\hline 1 & $180^{\circ}$ & 1 \\
1 & $90^{\circ}$ & 2 \\
2 & $180^{\circ}$ & 4 \\
2 & $90^{\circ}$ & 8 \\
\hline \hline
\end{tabular}

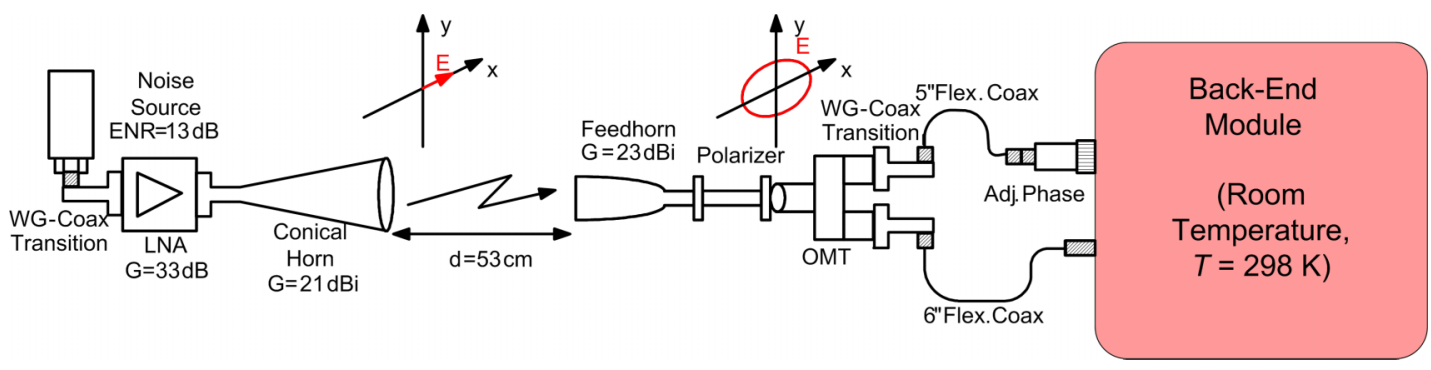

FIG. 7. Receiver functionality test bench (the back-end module corresponds to the room temperature part of the receiver in Fig. 1). 


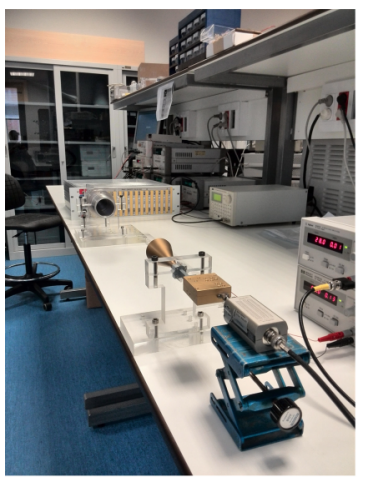

(a)

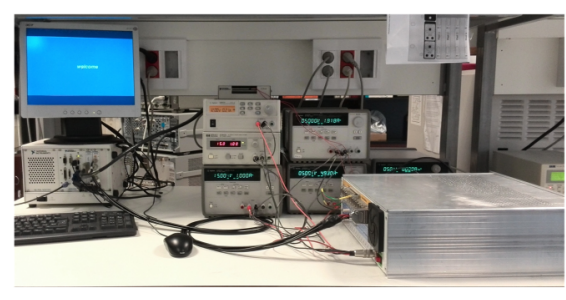

(b)

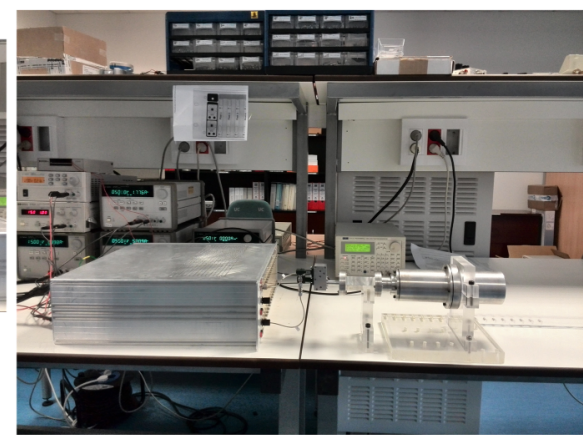

(c)

FIG. 8. Photograph of the polarimeter test bench. (a) View of the x-axis linearly polarized signal generator and the input feedhorn. (b) Rear view with the connection to the acquisition system. (c) Optomechanics and connection to back-end module input.

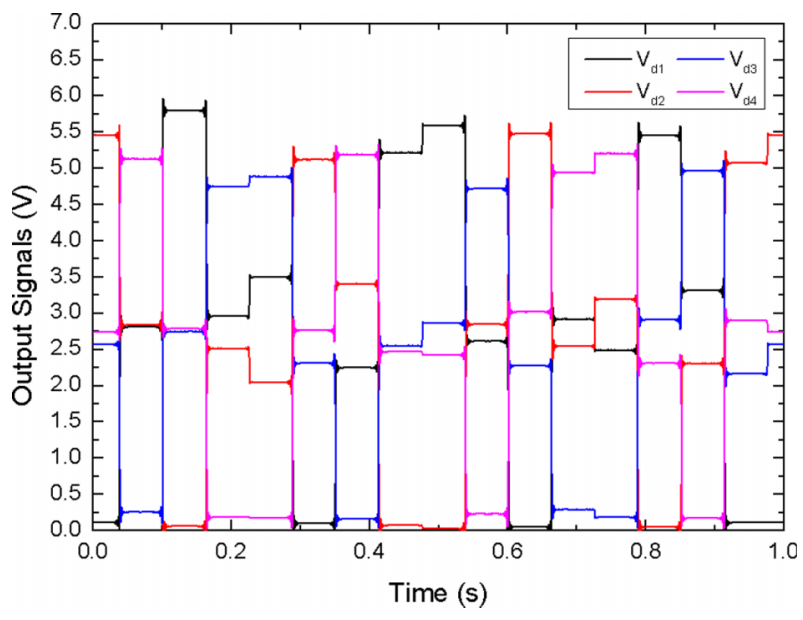

FIG. 9. Output signals of the receiver for an $\mathrm{x}$-axis linear polarization input signal.

each phase switch circuit and change the state of the receiver. The frequencies of each TTL signal and the phase switch which is activated using each one are listed in Table VI.

The tests at DICOM facilities are made using a PXI1031DC module from National Instruments as DAS in the setup, implementing the measurements under LabVIEW software with a 24-bits NI PCI/PXI-4462 card. The tests consist of measuring the detected voltages of the receiver in a defined period of time and the Stokes parameters are calculated after processing the values. A sampling rate of $1 \mathrm{kHz}$ is configured in the system and the number of points is equal to 1000, which corresponds to $1 \mathrm{~s}$ of acquisition time and real data. This period is the minimum needed time to cover a complete period of all the states of the phase switches with the TTL signals configured as in Table VI. The calculation method is based on the analysis of the values obtained from each detected voltage in each state and the comparison among them. The adjusting phase is configured to minimize the difference between the two mid-voltage outputs, corresponding to the values $(K / 2) \cdot A^{2}$ in Table II, in a concrete state. Then, the full sequence of states is analyzed calculating the parameters in each phase state.

TABLE VII. Average detected voltages in each output and each phase state.

\begin{tabular}{cccccccc}
\hline \hline State & $\Phi_{T}(\mathrm{deg})$ & $\Phi_{B 2}(\mathrm{deg})$ & $\Phi_{B 1}(\mathrm{deg})$ & $V_{d 1}$ & $V_{d 2}$ & $V_{d 3}$ & $V_{d 4}$ \\
\hline 0 & 0 & 0 & 0 & 5.589 & 0.019 & 2.861 & 2.424 \\
1 & 90 & 90 & 0 & 2.613 & 2.848 & 4.720 & 0.226 \\
2 & 180 & 180 & 0 & 0.049 & 5.484 & 2.274 & 3.017 \\
3 & 270 & 270 & 0 & 2.917 & 2.546 & 0.286 & 4.944 \\
4 & 270 & 0 & 90 & 2.487 & 3.193 & 0.179 & 5.204 \\
5 & 0 & 90 & 90 & 5.457 & 0.046 & 2.908 & 2.308 \\
6 & 90 & 180 & 90 & 3.312 & 2.305 & 4.966 & 0.167 \\
7 & 180 & 270 & 90 & 0.107 & 5.076 & 2.162 & 2.900 \\
8 & 180 & 0 & 180 & 0.113 & 5.456 & 2.566 & 2.742 \\
9 & 270 & 90 & 180 & 2.811 & 2.838 & 0.251 & 5.130 \\
10 & 0 & 180 & 180 & 5.800 & 0.131 & 2.745 & 2.786 \\
11 & 90 & 270 & 180 & 2.957 & 2.510 & 4.746 & 0.182 \\
12 & 90 & 0 & 270 & 3.496 & 2.044 & 4.887 & 0.175 \\
13 & 180 & 90 & 270 & 0.096 & 5.117 & 2.307 & 2.761 \\
14 & 270 & 180 & 270 & 2.245 & 3.401 & 0.159 & 5.187 \\
15 & 0 & 270 & 270 & 5.215 & 0.067 & 2.548 & 2.465 \\
\hline \hline
\end{tabular}


TABLE VIII. Stokes parameters and isolation ratios in each phase state.

\begin{tabular}{lccccc}
\hline \hline & & & & $\begin{array}{c}Q / I \\
(\mathrm{~dB})\end{array}$ & $\begin{array}{c}U / Q \\
(\mathrm{~dB})\end{array}$ \\
\hline$\Phi_{T}$ (deg) & $I$ & $Q$ & $U$ & -0.049 & -21.427 \\
0 (state \#10) & 5.732 & 5.669 & 0.041 & -12.804 \\
90 (state \#1) & 5.205 & 4.494 & 0.236 & -0.638 & -14.813 \\
180 (state \#8) & 5.439 & 5.343 & 0.175 & -0.078 & -22.577 \\
270 (state \#9) & 5.516 & 4.879 & 0.027 & -0.533 & - \\
\hline \hline
\end{tabular}

Once the Stokes parameters are obtained, two figures of merit are defined as the isolation between the $Q$ and $I$ parameters and the $U$ and $Q$ parameters, which are given by

$$
\begin{aligned}
Q /\left.I\right|_{d B} & =10 \cdot \log _{10}(Q / I), \\
U /\left.Q\right|_{d B} & =10 \cdot \log _{10}(U / Q) .
\end{aligned}
$$

Ideally, they should be 0 and $-\infty$ values, but in a real scenario, they denote the leakage signal due to the imperfections of the subsystems which are part of the receivers.

Since the phase adjust is only made for one state, a certain phase imbalance will affect to the others states. Therefore, from the analysis of the isolation ratios, a combination of four individual phase states $\left(\Phi_{T}=0^{\circ}, 90^{\circ}, 180^{\circ}\right.$, and $\left.270^{\circ}\right)$ achieving significant isolation performances defines a sequence of states for each pixel of the receiver in which outstanding results are expected. This is possible since the combination of the sixteen individual states of the phase switch module branches originates redundant phase states. This set of four different phase states is defined as the operation sequence for the analysed receiver chain. The TTL signals commanding the phase switches module states must be well known to determine the operation of the receiver chain. This procedure must be carried out for each pixel and individual state sequences for each one will be defined.

The test bench used for the test is shown in Fig. 8, in which a back-end rack, composed of sixteen receiver chains, is used to perform the measurements. The functionality test is performed in one of the pixel inside the back-end rack. The optomechanic components are connected through 2.92-mm coaxial cables to the back-end inputs, using WR28 waveguide to $2.4-\mathrm{mm}$ and $2.4-\mathrm{mm}$ to $2.92-\mathrm{mm}$ transitions. The detected voltages at the output of the pixel are measured in the PXI system and are shown in Fig. 9.

Each detected voltage $\left(V_{d 1}-V_{d 4}\right)$ is measured in the 16 phase states during a complete switching period time, and the average values for each phase state and detected voltage are listed in Table VII. They are listed in the phase order expected in the receiver according to the TTL control signals. Thus, the so-called state "0" corresponds to the eighth phase change in Fig. 9 in which the detected voltage $V_{d 1}$ gives the maximum value according to Table II. Then, a $90^{\circ}$-phase change in the receiver state makes that $V_{d 1}$ goes to medium voltage value while $V_{d 4}$ takes the minimum one. From these values, the Stokes parameters for the best phase states are obtained and they are listed in Table VIII. As the intensity Stokes parameter I can be calculated in two ways (see Table I), it is listed as the mean value between both of them.

\section{CONCLUSION}

The analysis, design, and characterization of the TGI receiver for the QUIJOTE instrument have been described. The theory background of the instrument is developed in order to obtain the Stokes parameters of an incoming electromagnetic signal, and the functionality of the receiver is analyzed. A room temperature test is performed using a wideband noise linearly polarized signal which is converted into a circular polarization signal by the system. A set of output detected signals are obtained which enable the calculations of the $I, Q$, and $U$ Stokes parameters, simultaneously, with significant isolation values. The results obtained validate the use of the proposed receiver scheme as a polarimeter aimed to characterize the CMB polarization.

\section{ACKNOWLEDGMENTS}

The authors would like to thank Spanish Ministry for Economy and Competitiveness for the financial support provided under the CONSOLIDER-INGENIO 2010 programme under Reference No. CSD2010-00064. The authors also thank Eva Cuerno, with the Departamento Ingeniería de Comunicaciones, and Santiago Cembellin, with the Instituto de Física de Cantabria, for their assistance in circuit and chassis assembly and manufacture.

${ }^{1}$ D. Cristian, Maps of Time: An Introduction to Big History (University of California Press, Berkeley, 2004).

${ }^{2}$ R. A. Alpher, H. Bethe, and G. Gamow, Phys. Rev. 73, 803 (1948).

${ }^{3}$ A. A. Penzias and R. W. Wilson, Astrophys. J. 142, 419 (1965).

${ }^{4}$ P. L. Richards, "Cosmic microwave background experiments — Past, present and future," in 32nd International Conference on Infrared and Millimeter Waves, 2007 and the 2007 15th International Conference on Terahertz Electronics, Cardiff, 2-9 September 2007 (IEEE, 2007), pp. 12-15.

${ }^{5}$ B. Aja, E. Artal, L. de la Fuente, J. P. Pascual, A. Mediavilla, N. Roddis, D. Kettle, W. F. Winder, L. Pradell, and P. de Paco, IEEE Trans. Microwave Theory Tech. 53, 2050 (2005).

${ }^{6}$ M. Kamionkowski, A. Kosowsky, and A. Stebbins, Phys. Rev. D 55, 7368 (1997).

${ }^{7}$ P. Kangaslahti, T. Gaier, M. Seiffert, S. Weinreb, D. Harding, D. Dawson, M. Soria, C. Lawrence, B. Hooberman, and A. Miller, "Planar polarimetry receivers for large imaging arrays at Q-band," in IEEE MTT-S International Microwave Symposium Digest, San Francisco, CA, 11-16 June 2006 (IEEE, 2006), p. 89.

${ }^{8}$ D. Kettle and N. Roddis, IEEE Trans. Microwave Theory Tech. 55, 2700 (2007).

${ }^{9}$ J. A. Rubiño-Martin, R. Rebolo, M. Tucci, R. Génova-Santos, S. R. Hildebrandt, R. Hoyland, J. M. Herreros, F. Gómez-Renasco, C. López, E. Martínez-González, P. Vielva, D. Herranz, F. J. Casas, E. Artal, B. Aja, L. de la Fuente, J. L. Cano, E. Villa, A. Mediavilla, J. P. Pascual, L. Piccirillo, B. Maffei, G. Pisano, R. A. Watson, R. Davis, R. Davies, R. Battye, R. Saunders, K. Grainge, P. Scott, M. Hobson, A. Lasenby, G. Murga, C. Gómez, A. Gómez, J. Arino, R. Sanquirce, J. Pan, A. Vizcarguenaga, and B. Etxeita, in Highlights of Spanish Astrophysics V, Astrophysics and Space Science Proceedings, Part 3 (Springer, Berlin, 2010), pp. 127-135.

${ }^{10}$ M. López-Caniego et al., "Instrumentation and methods for astrophysics," in Conference Proceedings Rencontres du Vietnam, Quy Nhon, Vietnam, 28 July-3 August 2013, p. 1.

${ }^{11}$ E. Collet, Field Guide to Polarization (SPIE Press, Bellingham, 2005).

${ }^{12}$ J. A. Rubiño-Martin et al., Proc. SPIE 8444, 84442Y (2012).

${ }^{13}$ J. L. Cano, E. Villa, J. Cagigas, B. Aja, J. V. Terán, A. R. Pérez, L. de la Fuente, E. Artal, A. Mediavilla, and R. Hoyland, "Multi-pixel Ka-band radiometer for the QUIJOTE experiment (Phase II)," in 42nd European Microwave Conference (EuMC), Amsterdam, 29 October-1 November 2012 (IEEE, 2012), pp. 37-40. 
${ }^{14}$ W. Zhong, B. Li, Q. Fan, and Z. Shen, "X-band compact septum polarizer design," in IEEE International Conference on Microwave Technology and Computational Electromagnetics, Beijing, 22-25 May 2011 (IEEE, 2011), pp. 167-170.

${ }^{15}$ J. L. Cano, E. Villa, B. Aja, L. de la Fuente, E. Artal, R. Watson, E. Blackhurst, J. Edgley, and C. Baines, "The Ka-band receiver for the
QUIJOTE experiment," in 6th European Microwave Integrated Circuits Conference, Manchester, 10-11 October 2011 (IEEE, 2011), pp. 620-623. ${ }^{16}$ E. Villa, B. Aja, L. de la Fuente, and E. Artal, Electron. Lett. 45, 556 (2009).

${ }^{17}$ E. Villa, B. Aja, J. Cagigas, L. de la Fuente, and E. Artal, Rev. Sci. Instrum. 84, 124704 (2013).

${ }^{18}$ E. Villa, Ph.D. thesis, Universidad de Cantabria, Spain, 2014. 